Supporting Information

\title{
Combining Gemcitabine-loaded macrophage-like nanoparticles and Erlotinib for pancreatic cancer therapy
}

\author{
Hongqiao Cai ${ }^{1}$, Ruobing Wang ${ }^{1}$, Xingren $\mathrm{Guo}^{1}$, Meiyu Song ${ }^{2}$, Fei Yan ${ }^{2}, \mathrm{Bai}^{\mathrm{Ji}}{ }^{1 *}$,
} Yahui Liu ${ }^{1 *}$

1 Department of Hepatobiliary and Pancreatic Surgery, the First Hospital, Jilin University, 71 Xinmin Street, Changchun 130021, China

2 State Key Laboratory of Inorganic Synthesis and Preparative Chemistry, International Joint Research Laboratory of Nano-Micro Architecture Chemistry (NMAC), International Research Center for Chemistry-Medicine Joint Innovation, College of Chemistry, Jilin University, 2699 Qianjin street, Changchun 130012, China Corresponding author:

Yahui Liu (E-mails: yahui@jlu.edu.cn; Tel: 86-13009109056)

Bai Ji (E-mails: ji_bai@jlu.edu.cn; Tel: 86-13604334640) 


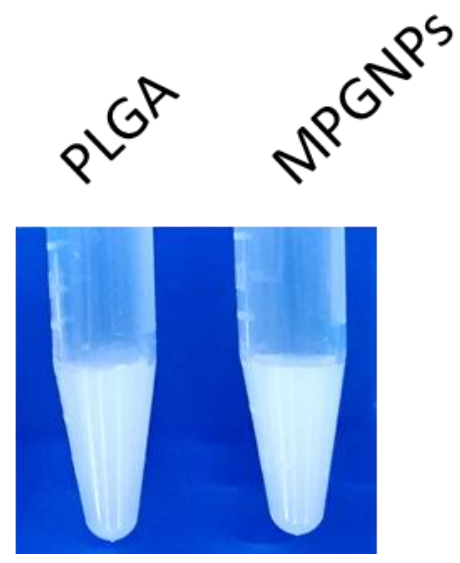

Figure S1. The photographs of PLGA and MPGNPs. 

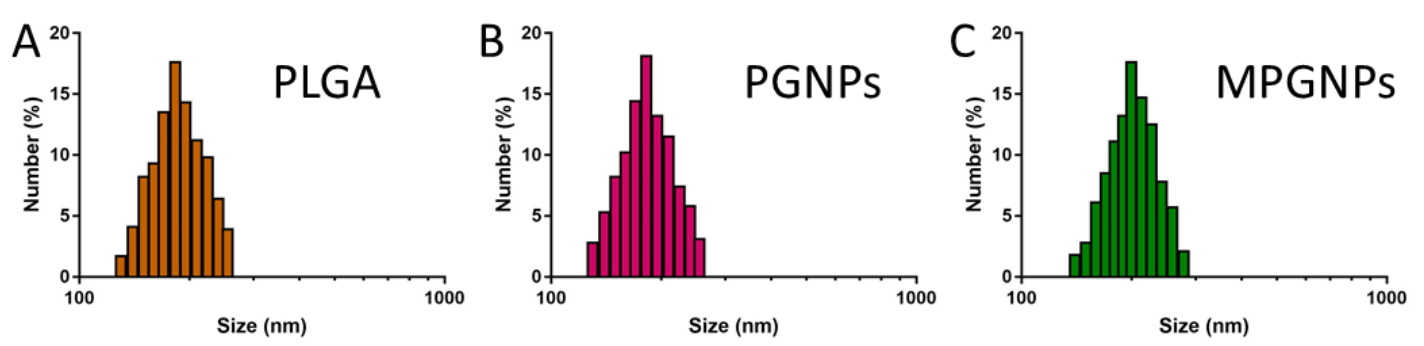

Figure S2. The particle size distribution of PLGA (A), PGNPs (B) and MPGNPs (C). 


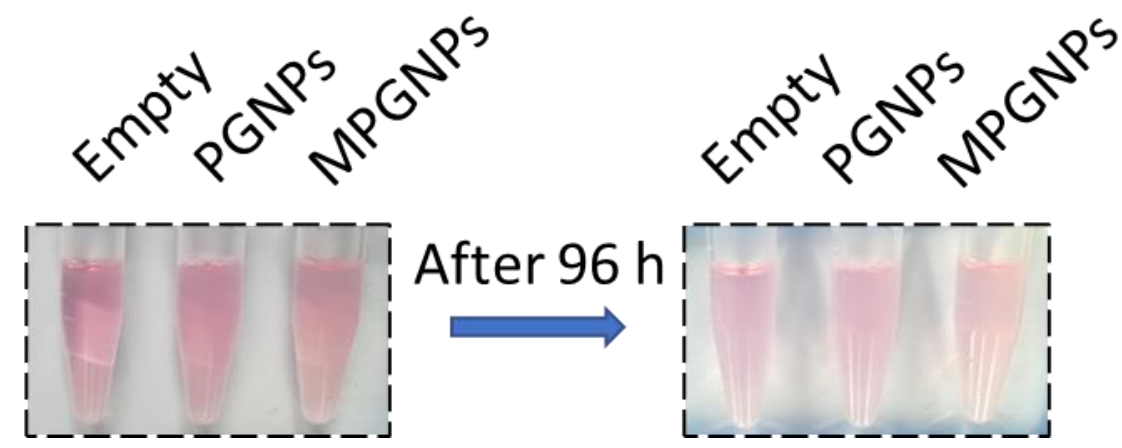

Figure S3. The photographs of empty cell culture medium supplemented with $10 \% \mathrm{FBS}$, medium with PGNPs and medium with MPGNPs at $0 \mathrm{~h}$ and $96 \mathrm{~h}$. 


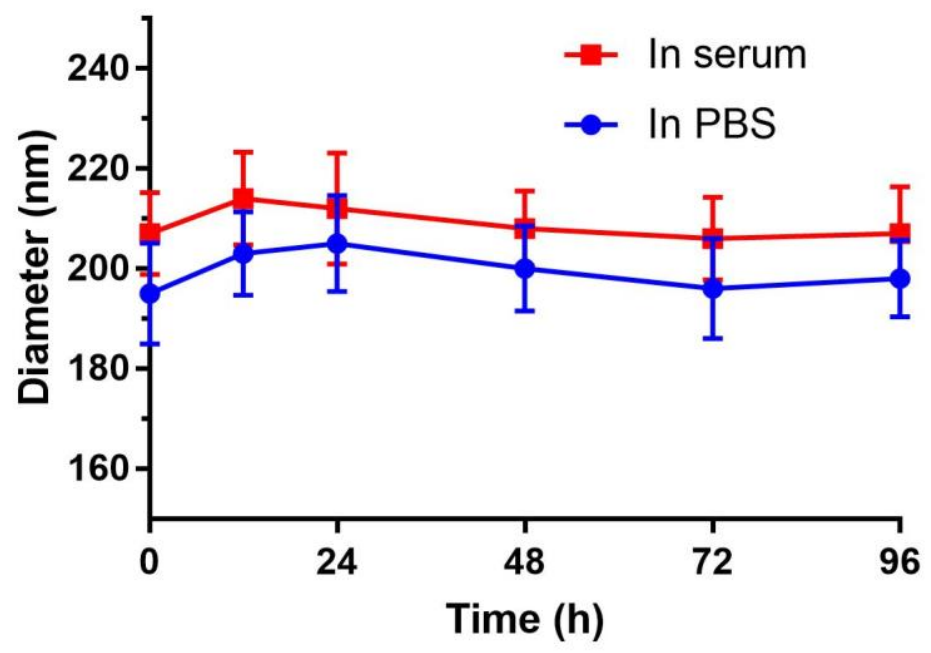

Figure S4. The particle size of MPGNPs incubated in PBS or serum from $0 \mathrm{~h}$ to $96 \mathrm{~h}$ 


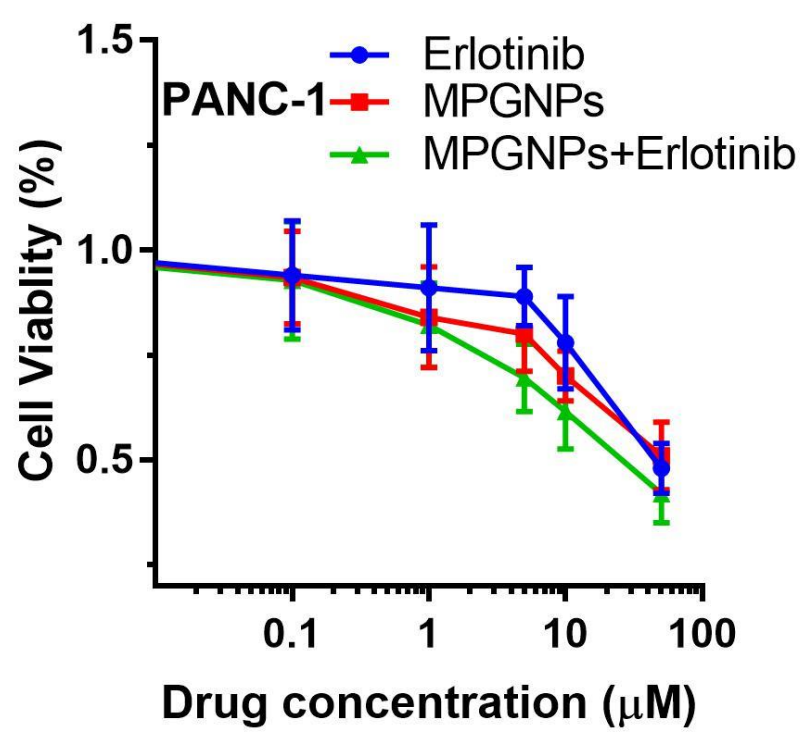

Figure S5. The cell viability of PANC-1 cells treated with Erlotinib, MPGNPs or combo after $72 \mathrm{~h}$. 


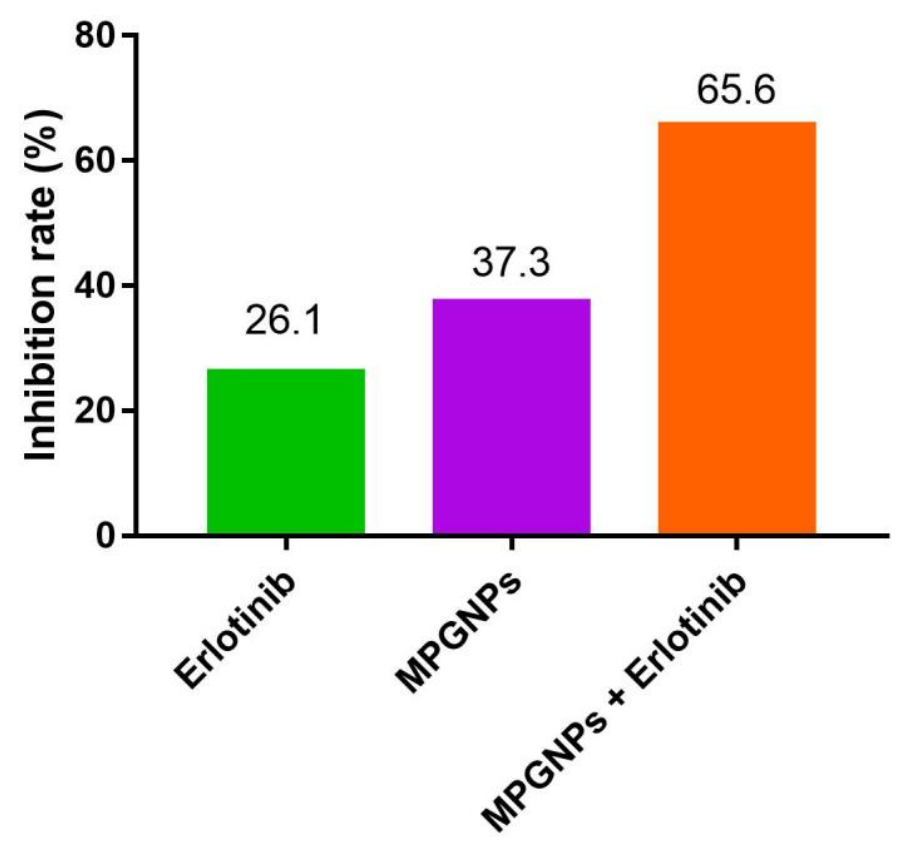

Figure S6. The inhibition rate of Erlotinib, MPGNPs, and MPGNPs + Erlotinib 


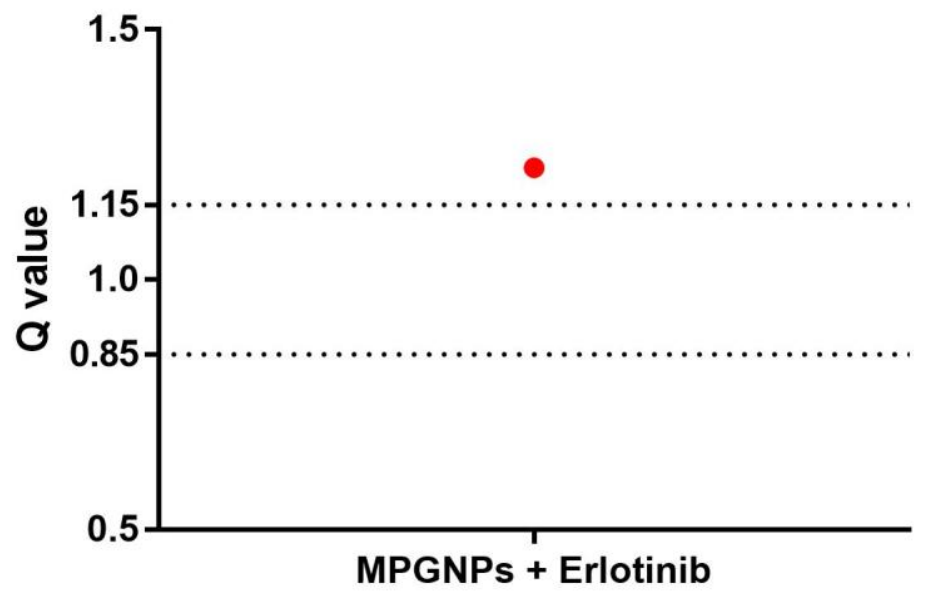

Figure S7. Q value of MPGNPs + Erlotinib.

$(\mathrm{Q}<0.85$, reveals the antagonism effect; $0.85 \leq \mathrm{Q}<1.15$, reveals the additive effect; $1.15 \leq \mathrm{Q}$, reveals the synergistic interaction) 


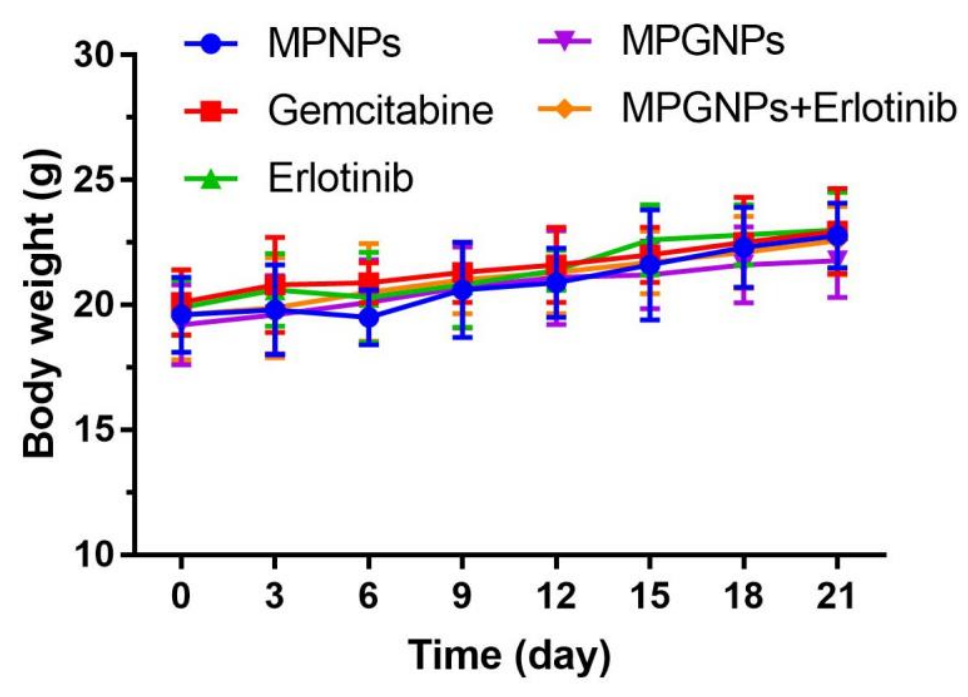

Figure S8. The body weight of mice treated with MPNPs, Gemcitabine, Erlotinib, MPGNPs, and MPGNPs combined with Erlotinib. 


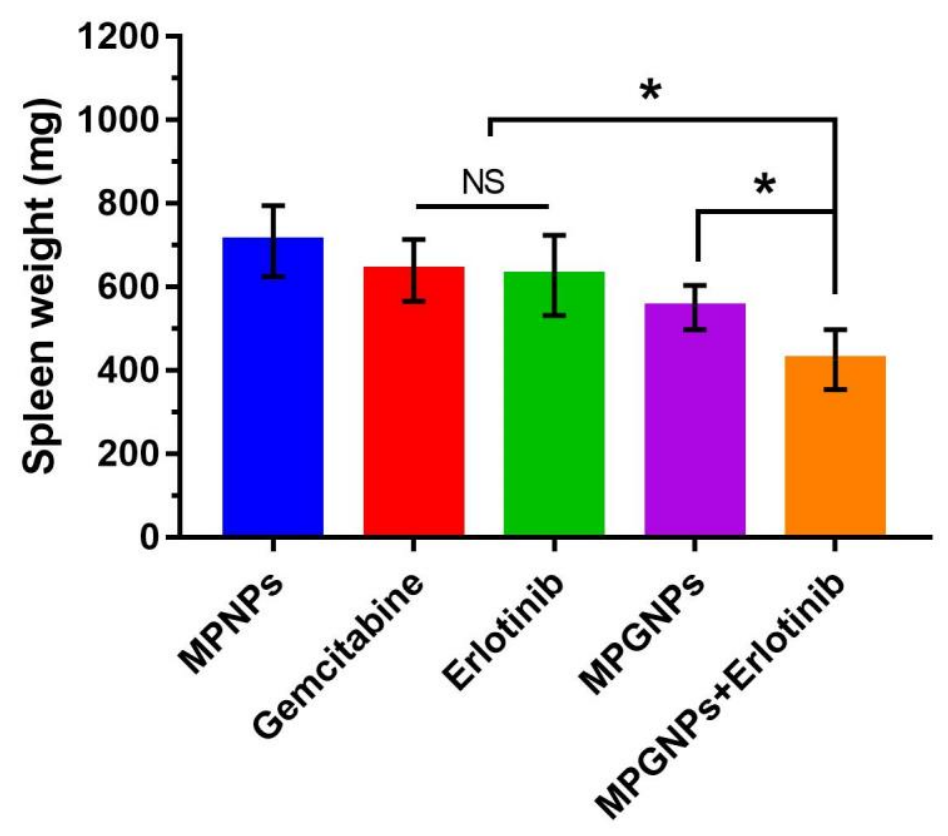

Figure S9. The spleen weight of mice from each group. NS, no significance; $* \mathrm{P}<0.05$ 


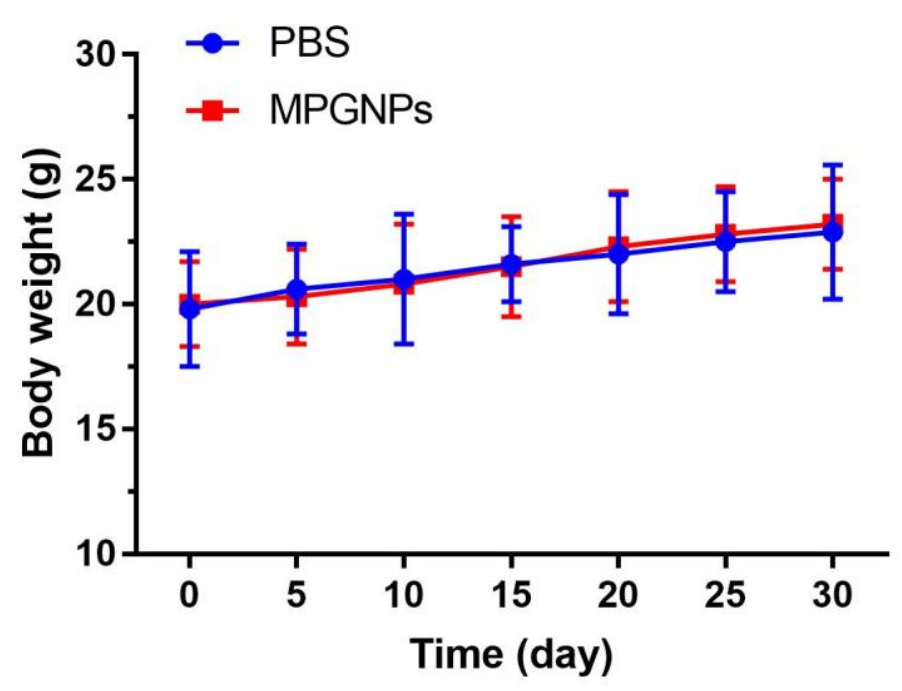

Figure S10. The body weight of normal mice (with no tumor inoculation) treated with PBS and MPGNPs $(n=3)$. 


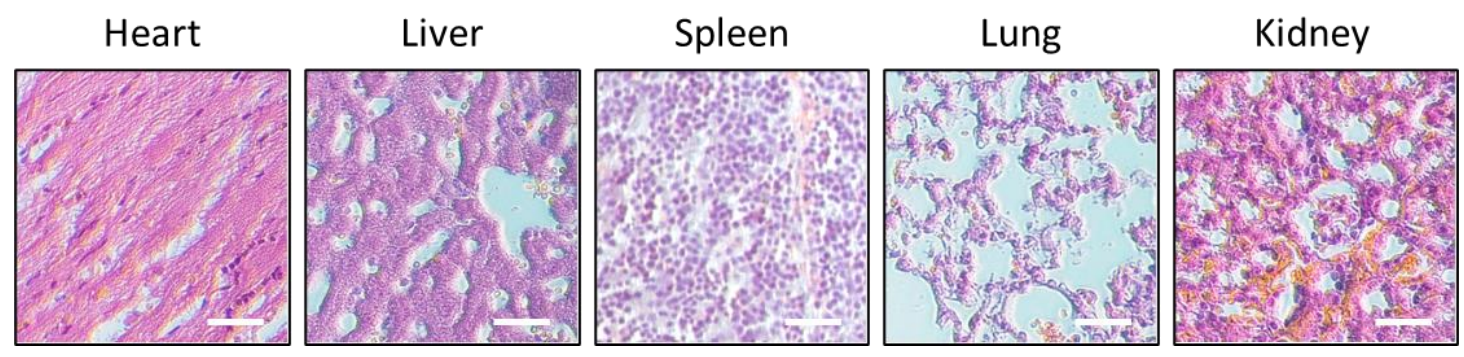

Figure S11. Representative images of H\&E-stained tissue sections of mice treated with MPGNPs. Scale bar $=50 \mu \mathrm{m}$. 